\title{
Evaluating Mental Health in Cuban Refugees: The Role of the Refugee Health Screener-15
}

\author{
Rahel S. Bosson, MD;† Monnica T. Williams, PhD; Victoria A. Schlaudt, MS; Ruth M. Carrico, PhD, FNP-C; Adriana Peña; \\ Stanley Kotey, MB, ChB, MPH; Julio A. Ramirez, MD; Jonathan Kanter, PhD
}

\begin{abstract}
The Refugee Health Screener-15 (RHS-15) is widely used in refugee populations, but the psychometric properties and clinical utility have not been evaluated in Cuban refugees and entrants. The current study explored results from the Spanish version of the RHS-15 in a sample of 53 Cuban entrants and refugees, and of these, $17.6 \%$ screened positive for a mental health concern. Analyses suggested that a positive screening was significantly related to symptoms of anxiety, depression, and posttraumatic stress disorder; however, it was not associated with demographic variables such as gender, mode of transport, or the number of countries through which a refugee traveled before arriving in the United States. A factor analysis of the measure revealed a 4factor solution is most appropriate when used with Cuban individuals. Results provide preliminary evidence regarding the measure's psychometric properties, and demonstrate that the Spanish version of the RHS-15 is an appropriate screening tool for the mental health of newly arrived Cuban entrants and refugees. Future studies should further validate the Spanish version RHS15 in Cuban entrants and refugees, and explore its efficacy amongst Latino refugees of other nationalities.
\end{abstract}

DOI: $10.18297 /$ rgh/vol1/iss $1 / 4$
Website: https://ir.library.louisville.edu/rgh
Affiliations:
University of Louisville Global Health Program,
Division of Infectious Diseases, University of
Louisville School of Medicine, Louisville, KY USA
(RSB, RMC, SK, JAR)
University of Connecticut, Department of Psy-
chological Sciences, Storrs, CT (MTW)
Nova Southeastern University, College of Psy-
chology, Fort Lauderdale, FL (VAS)
Spalding University, Department of Psychology,
Louisville, KY (AP)
University of Washington, Department of Psy-
chology, Seattle, WA (JK)
(C) 2017, The Author(s).
CC (i)

\section{Introduction}

Recent estimates from the United Nations High Commissioner for Refugees (UNHCR) state that there are currently 24.5 million refugees and asylum-seekers in the world (UNHCR 2015). A refugee is defined as a person who "has been forced to flee his or her country because of persecution, war, or violence" and "has a well-founded fear of persecution for reasons of race, religion, nationality, political opinion or membership in a particular social group" (UN General Assembly 1951). Cubans often enter the US as an asylum applicant (asylee) or one who has been granted an entrant status where they are temporarily allowed to enter the US for humanitarian, medical or legal reasons (Aspinall and Watters 2010). Entrants are included in the refugee resettlement programs and those individuals are eligible for assistance through

\footnotetext{
Correspondence To: Rahel S. Bosson, MD

Assistant Professor and Medical Director

University of Louisville Global Health Program

Division of Infectious Diseases

University of Louisville School of Medicine

501 E Broadway, Suite 140C

Louisville, KY, USA, 40202

Phone: 502-217-1072

Email: rahel.bosson@louisville.edu
}

resettlement agencies including an initial physical examination, also called the domestic health screen. This examination includes physical and mental health screenings with the purpose of identifying current health issues and is a bridge to primary and specialty care needs that may be important as the refugee begins the journey of self-sufficiency and citizenship (CDC 2012a; 2012b). Entrants and refugees have experienced significant adversity and were potential victims of traumatizing events. This population, therefore, experiences higher levels of mental health difficulties, including anxiety, depression, and posttraumatic stress disorder (PTSD), in comparison to non-entrants and non-refugees (Pumariega, Rothe, and Pumariega 2005).

There has been a recent surge in the number of Cubans entrants and refugees arriving to the US. In fact, during the first ten months of 2016, approximately 46,635 Cubans entered the US, an increase from a total of 43,159 in 2015 and 24,278 in 2014 (Krogstad 2016). Cuban refugees, in particular, have demonstrated high rates of depressive disorders, anxiety, substance use, and psychosis (Portes, Kyle, and Eaton 1992). In addition to the steep increase in Cuban entrants, the US accepts thousands of Spanish-speaking refugees from a variety of other countries each year. According to the Bureau of Population, Refugees, and Mi- 
gration, the US accepted 31,200 Spanish-speaking refugees between 2008 and 2016 in addition to the aforementioned Cubans (Refugee Processing Center 2016). Experts in the field have stated that entrants and refugees are at risk for several reasons including arduous and dangerous travel through many countries to arrive to the US, uncertainty about immigration status, and an exacerbation of previous medical and mental health issues due to the stress and upheaval they experience (Ventevogel et al. 2015), in addition to the multiple stressors and risks faced by Spanishspeaking immigrants to the US, regardless of refugee status, such as language barriers, acculturative stress, and loss of family and other support resources (Kirmayer et al. 2011). Thus, there is a need for measures that can assess the mental health status of incoming Spanish-speaking refugees and entrants in order to guide clinical research and intervention. Further, this information will allow mental health professionals to efficiently screen a large number of refugees and entrants for psychopathology using a standardized approach in order to facilitate appropriate care.

Challenges exist in providing mental health screening for all incoming refugees as it involves a process best accomplished with expert and culturally tailored guidance. When questioned about mental health screening practices, 19 out of 44 state refugee health coordinators expressed that they did not conduct any mental health screening for incoming refugees, and of those who performed a screening, 70.8\% used an informal conversation to assess psychopathology (Slonim-Nevo and Regev 2016). Recognizing the need for a standardized and efficient approach to refugee mental health screening that assessed a wide range of symptoms (Pathways to Wellness 2012), Hollifield and colleagues created the Refugee Health Screener-15 (RHS-15) to screen refugees for anxiety, depression, and PTSD (Hollifield et al. 2013). It was developed with items similar to those in pre-existing validated measures such as the New Mexico Refugee Symptom Checklist-121 (Hollifield et al. 2009), Hopkins Symptom Checklist-25 (Derogatis et al. 1974), and Posttraumatic Symptom Scale-Self Report (Parloff, Kelman, and Frank 1954).

The RHS-15 was validated using a sample of 250 refugees from Burma, Bhutan, and Iraq who spoke Nepali, Karen, Burmese, and Arabic languages. In the initial development study for the RHS15, Hollifield and colleagues (2013) reported that of their 77 (30.7\%) positive screens with the RHS-15, 74\% accepted a referral for treatment and 5\% had already sought treatment. At the time of the measure's creation, Burmese, Bhutanese, and Iraqis were the most common incoming groups of refugees; thus, the measure's validation sample filled a vital gap in refugee arrival services (Hollifield et al. 2013; Hollifield et al. 2016). Since that time, the number of Cuban refugees and asylum-seekers has grown, demonstrating the necessity of a validated Spanish version of this measure.

The purpose of the current study is to perform a preliminary investigation of the Spanish version of the RHS-15 to evaluate its psychometric properties when used with Cuban entrants and refugees and to provide a general overview of the mental health of this population. The results of this study may help to determine if the RHS-15 is appropriate for use with this vulnerable population, and can serve as a starting point for future larger studies to further validate this measure and evaluate the mental health of Cuban entrants and refugees.

\section{Methods}

For more than twenty years, the Division of Infectious Diseases at the University of Louisville has been providing care for vulnerable populations with special needs, as well as outreach programs for the Louisville community. In response to significant growth, services were consolidated into the University of Louisville Global Health Center (UL-GHC) in 2014. At present, care is provided in the UL-GHC for the more than 3,000 refugees resettling in the area each year, with Cubans representing the largest population. As part of the resettlement process, refugees/entrants are eligible for an initial health screening. The goal is to complete this screening within the first 90 days of arrival to the US and the UL-GHC is one of two sites for provision of this health screening for refugees and other entrants resettling in Louisville, Kentucky. During the health screening process, patients also receive mental health screening. Fifty-three Cuban refugees/entrants presenting for that initial health screening in 2016 were included in the study following review and approval by the University of Louisville Institutional Review Board.

Most of the participants identified as entrants $(\mathrm{N}=46 ; 87 \%)$ and the remainder identified as refugees. Most indicated that Spanish was their preferred language $(\mathrm{N}=51 ; 96 \%)$, and the rest were bilingual. The vast majority of participants did not report a mental health disorder documented prior to their arrival $(\mathrm{N}=51$; $96 \%)$. The mean number of countries traversed on the trip from Cuba to the US was $4.60(S D=2.70)$. See Table 1 for additional demographic details.

\section{Procedures}

Each participant was administered the RHS-15 and a battery of related measures by a doctoral-level student evaluator. A total of 4 evaluators conducted evaluations and 2 of these were native bilingual Spanish-speakers. Participants with non-Spanish speaking evaluator were provided with an interpreter (15.1\%). All evaluators were trained in cultural competence and provided ongoing supervision for their work with this population in a weekly group meeting (supervised by MTW and JK). Information about confidentiality and privacy was provided to all participants, which included a summary form of this information in Spanish. For participants who were illiterate, the confidentiality form and all questionnaires were read to them. Participants indicating mental health concerns were offered on-site therapy at no charge.

Clinical measures that were not available in Spanish were translated as needed by native bilingual Spanish-speaking clinicians. Translation procedures for these measures included initial translation of English materials into Spanish by study clinicians, and then back-translation by an additional bilingual research assistant. The participants who were screened were selected at random and were provided with information about the screening 
process. Participants were asked for their consent and were given the option to revoke the consent at any time. The screenings were generally done in one session which lasted between one and four hours; however, some participants requested to complete the screening in two sessions. Additional information about the development of the clinic and procedures utilized is available elsewhere (Bosson et al. 2017).

\section{Measures}

Refugee Health Screener-15. The RHS-15 is a 15-item measure that assesses refugee mental health (Hollifield et al. 2013). It is comprised of 13 items regarding symptoms, one item regarding coping, and one distress thermometer. Symptoms are rated for how bothersome they have been in the past month on a Likert type scale from 0 (not at all) to 4 (extremely). The item used to assess coping is a general question regarding ability to cope with "whatever comes" and is answered on a Likert type scale from 0 (able to cope with anything) to 4 (able to cope with nothing). A positive diagnosis from the RHS-15 is defined as a total score of 12 or above on the first 14 items and/or a score of 5 or greater on the distress thermometer (range from 0 to 10). Excellent reliability was found in two validation studies for the RHS-15, which were conducted in the Karen, Burmese, Nepali, and Arabic languages ( $\alpha=.92$ to .95; Hollifield et al. 2013; Hollifield et al. 2016). Validity of the measure was demonstrated through strong positive correlations with measures of anxiety, depression, and PTSD. Further, an RHS-15 diagnosis and total score both predict anxiety, depression, and PTSD (Hollifield et al. 2013; Hollifield et al. 2016).

Beck Anxiety Inventory (BAI). The Beck Anxiety Inventory (BAI; Beck 1990) is a widely used instrument designed to quantify levels of anxiety and is also useful in discriminating anxiety from depression. The scale consists of 21-items, each describing a common symptom of anxiety. Scores from 0-9 indicate minimal anxiety, 10-16 mild anxiety, 17-29 moderate anxiety, and 30-63 severe anxiety. A Spanish version of the BAI has been validated as well (Magán, Sanz, and García-Vera 2008). The reliability of the BAI in the current study was good $(\alpha=.896)$.

Beck Depression Inventory-II (BDI-II). The Beck Depression Inventory-II (BDI-II; Beck, Steer, and Brown 1996) is a widely used 21-item self-report measure of depressive symptoms. Interpretation of BDI-II scores is as follows: 0-13 minimal depression, 14-19 mild depression, 20-28 moderate depression, 29-63 severe depression. The Spanish version of the BDI-II has been validated for use (Penley, Wiebe, and Nwosu 2003). Reliability of the BDI-II in the current study was good $(\alpha=.891)$.

Hopkins Symptom Checklist-25 (HSCL-25). The HSCL-25 (Parloff, Kelman, and Frank 1954) is a well-known instrument used to screen for psychopathology, specifically anxiety and depression. A mean item score of 1.75 is used as the clinical cut off. This measure was translated into Spanish for this investigation. The HSCL-25 demonstrated excellent reliability for the current study $(\alpha=$.909).
Modified PTSD Symptom Scale (MPSS). The MPSS-SR is a 17item measure that assesses the frequency and severity of PTSD symptoms (Falsetti et al. 1993). Cut-off scores for community samples are 46 and treatment samples 71 for the combined scales. This measure was translated into Spanish for the current study. The full scale ( $\alpha=.886)$ and severity scale $(\alpha=.804)$ demonstrated good reliability in the current study; however, the frequency scale alone demonstrated a lower level of reliability ( $\alpha$ $=.668)$.

Posttraumatic Stress Checklist-Civilian Edition (PCL-5). The PCL-5 Civilian Edition (Weathers et al. 2013) is a widely used 17-item measure designed to assess posttraumatic stress symptoms in non-military populations. Preliminary validation on the measure indicates a cut-point of 33, with a lower cutoff to be considered when screening to maximize detection of possible cases. A Spanish version of previous versions of this measure has been validated for use (Marshall 2004; Orlando and Marshall 2002). However, the PCL-5 does not currently have a validated Spanish version. The reliability of this measure in the current study was excellent ( $\alpha=.910)$.

Demographics Questionnaire. The demographics questionnaire is a self-report measure created to assist with the screening process for refugees. The items ask about gender, race, age, education, previous or current occupations, children, income, religion, and history of mental illness outside of the United States. Participants were also queried about mode of transport to the US and the number of countries through which the refugee traveled before arriving in the United States.

\section{Analysis Plan}

Means, standard deviations, and frequency counts were conducted for the demographic variables and clinical measures. Differences in demographic and psychopathology variables between positive and negative screenings were analyzed with t-tests for continuous variables, Mann Whitney $\mathrm{U}$ tests for variables lacking normal distribution, and chi-square for categorical variables.

A principal component analysis (PCA) with the 15 items was conducted to determine the factor structure of the RHS-15 in the Cuban sample. Specifically, we employed the principal components extraction method with Varimax rotation, determining the number of factors to extract based on eigenvalues of over 1 and a visual inspection of the scree plot. Items were included on individual components if their loadings were .55 or higher (Costello and Osborne 2005). Pearson's correlations were used to examine the relationship between psychopathology measures and the total (14-item) RHS-15 score and the RHS-15 distress thermometer.

\section{Results}

\section{Descriptives}

Of the Cuban refugee/entrants participating, 18\% $(n=9)$ screened positive on the RHS-15. Total RHS-15 mean scores differed significantly between patients who screened RHS-negative and those who screened RHS-positive. There were no differ- 
ences amongst demographic variables between positive and negative screens on the RHS-15, including patient age, gender, use of a translator, having children, number of dependents in the US, method of transport to the US, monthly income, religion, marital status, education level, monthly income, immigration status, and number of countries traversed in route to the US.

When examining specific symptoms via individual RHS-15 items, mean differences were statistically significant for RHS-15 items "Muscle, bone and joints", "Feeling down, sad or blue", "Too much thinking/thoughts", "Crying easily", "Ability to cope" and the "Distress Thermometer". Borderline significant differences were however observed for "Feeling helpless" and "Jumpier, more easily startled" (Table 2). Two items that were infrequently endorsed by either group were the only two items, which referenced "the trauma" (i.e. "reliving the trauma" and physical reactions "when reminded of the trauma").

\section{Factor Structure}

Despite a less than optimal subject to item ratio for the PCA, the Kaiser-Meyer-Olkin (KMO) Test for Sampling Accuracy rated the sample at .58 (an adequate rating) and Bartlett's Test of Sphericity was highly significant $(p<.001)$, thus the PCA was deemed acceptable. Examination of eigenvalues over one and visual inspection of the scree plot suggested a four-component 10 solution, which explained $62.3 \%$ of variance. Inspection of item loadings suggested that the four components represented: (1) Weakness (7 items); (2) Health Symptoms (3 items); (3) Distressing Thoughts (2 items); and (4) Emotional Distress (2 items), as shown in Table 3. Although the majority of items had loaded at or above .55 on their respective component, one did not ("feeling sad, down, or blue most of the time"). Additionally, several items showed substantial cross-loading.

\section{Validity}

All clinical measures were significantly higher among those who screened positive on the RHS-15, (BDI-II Mann Whitney $\mathrm{U}=$ 27.00, $p<.01$; BAI Mann Whitney $\mathrm{U}=17.00$, $\mathrm{p}<.01$; HSCL-25 Mann Whitney $\mathrm{U}=48.50, p<.01$; PCL-5 Mann Whitney $\mathrm{U}=$ 32.00, $p<.01$; MPSS Mann Whitney $U=16.50, p<.01$ ). See Figure 1 for box plots of the variables of interest.

Pearson's correlations were conducted to assess the relationship of the variables of interest with the RHS-15 total score and distress thermometer. The RHS-15 total score and the distress thermometer were both correlated with anxiety scores on the BAI, depression symptoms from the BDI-II, posttraumatic stress symptoms identified in the PCL-5, and the internalizing symptoms from the HSCL-25. See Table 4.

\section{Discussion}

\section{Psychometric Properties}

When Hollifield and colleagues (2013) initially developed the RHS-15, they stated that there was a need for future studies to generalize the use of the RHS-15 to other ethnicities and languages in order to fill the gaps in our current refugee screening capacity. The current study aims to fill part of that gap by providing preliminary evidence of the psychometric properties of the Spanish version of the RHS-15 in a population of Cuban entrants/refugees.

With regards to the factor structure of the RHS-15, the current study revealed a 4-factor solution for the measure. One previous study (Hollifield et al. 2016) demonstrated mixed results regarding the factor structure of the measure. Overall, the authors asserted that a one factor solution emerged; however, they reported significant group differences. For example, Burmese individuals responded in a way that was consistent with a 4-factor solution, similarly to the current study. Contrastingly, Nepalese individuals responded in a way that was consistent with 1 or 2 factors, and 1 factor solution was best for Iraqi individuals. The previous study, along with the results of the current investigation, demonstrate that culture plays a role in the manner in which individuals respond to items on the RHS-15. More research is necessary to clarify and replicate these results in order to provide a deeper understanding of the measure's factor structure across cultures.

Items related to "the trauma" were hardly endorsed by the current study's sample, regardless of screening positive or negative. This could be due to the wording of the measure, which implies trauma has occurred, which may not be consistent with how most Cuban entrants/refugees perceive their journey and experiences. Furthermore, not endorsing the experience as traumatic may reflect stoicism in response to adversity, which is valued in Hispanic culture (Fierros and Smith 2006). This stoicism may be a factor in the low anxiety and depression scores endorsed by the refugees. It may be beneficial to either reword these items or remove them altogether from the Cuban version of the RHS-15 given the way Cubans may interpret their experiences. In addition, the lower levels of mental health difficulties endorsed by this sample may reflect the tendency to underreport psychological symptoms in Latino culture (Fierros and Smith 2006) and concern regarding the stigma that this group may face if they choose to disclose these symptoms (Kirmayer et al. 2011).

\section{Clinical Findings and Utility}

The significant differences on clinical measures of anxiety, depression, PTSD, distress, and the RHS-15 total score between positive and negative screenings on the RHS-15 demonstrates its clinical usefulness and ability to identify those experiencing significant psychopathology in Cuban entrants/refugees. The Spanish version of the RHS-15 demonstrated convergent validity through its strong correlations with anxiety, depression, internalizing symptoms and PTSD symptoms. It also showed discriminant validity through its lack of association with demographic variables. Thus, the Spanish version of the RHS-15 has preliminary evidence of validity when used with Cuban entrants/refugees.

About $17.6 \%$ of the Cuban entrants/refugees screened positive, which is much lower than the $30.7 \%$ who screened positive in the 

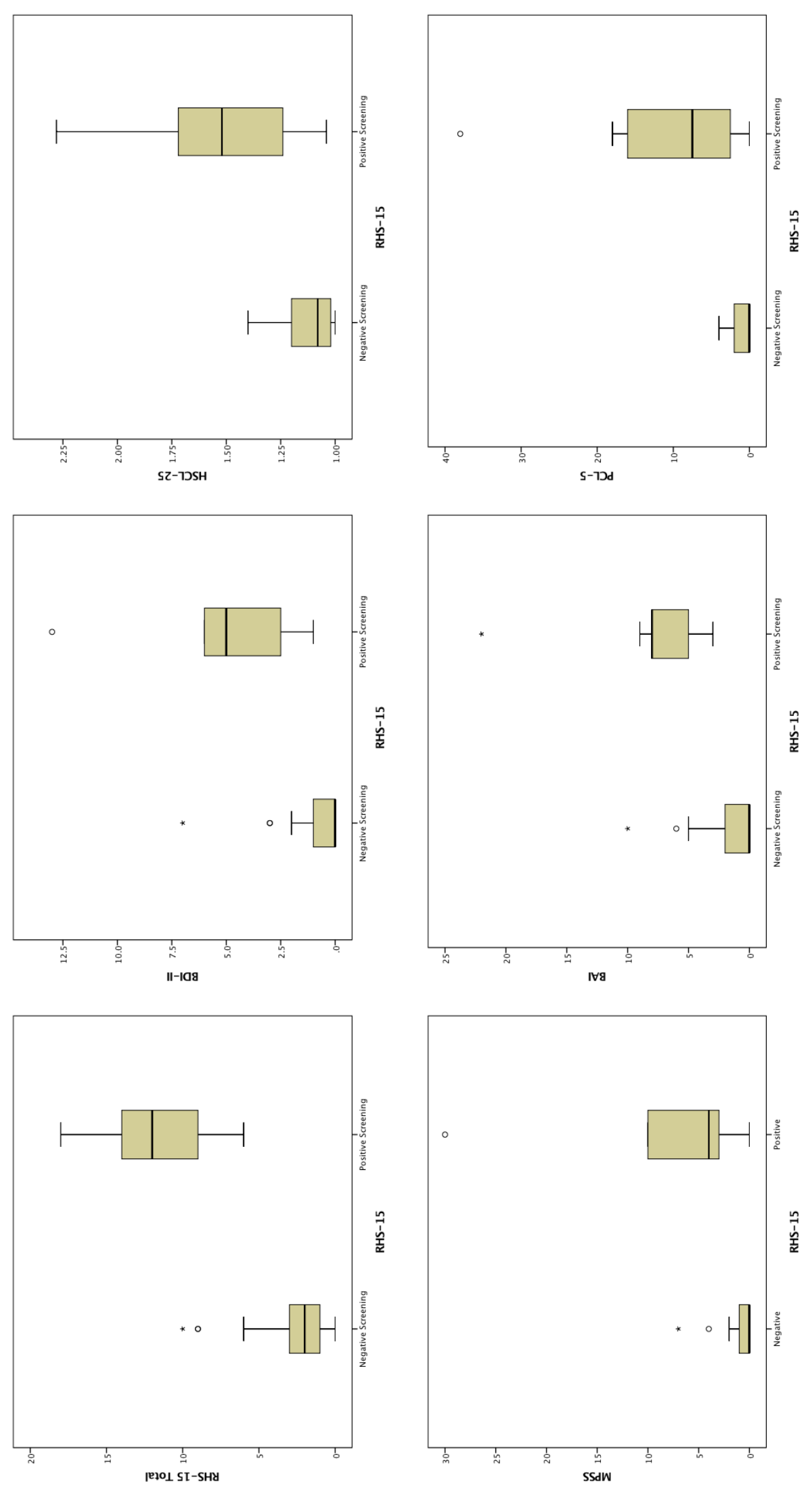

$\frac{\hat{h}}{\frac{1}{x}}$

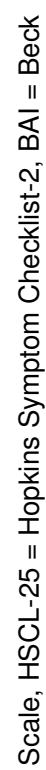

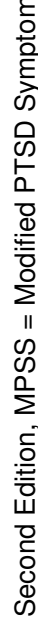

ํํำ

ฏ

을 ․ㅡㄹ

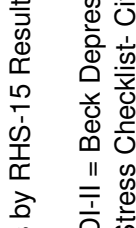

क ळे

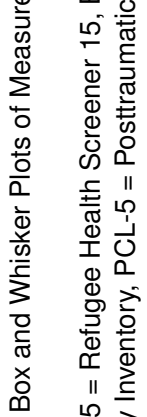

- $\quad \frac{1}{\frac{1}{2}}$

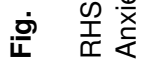


Table 1 Demographic variables

\begin{tabular}{lc}
\hline Demographic Variable & Frequency (Valid Percentage) \\
\hline Gender & $29(54.7 \%)$ \\
Male & $24(45.3 \%)$ \\
Female & $8(19 \%)$ \\
Religion & $5(11.9 \%)$ \\
Catholic & $19(45.2 \%)$ \\
Protestant & $10(23.8 \%)$ \\
Non-religious & \\
Other & $27(52.9 \%)$ \\
Marital Status & $17(33.3 \%)$ \\
Single & $7(13.7 \%)$ \\
Married & \\
Other & $27(55.1 \%)$ \\
Children & $22(44.9 \%)$ \\
Yes & \\
No & $42(85.7 \%)$ \\
Immigration Status & $7(14.3 \%)$ \\
Entrant/Parolee & \\
Refugee & $1(3.4 \%)$ \\
Pre-Migration Diagnosis & $28(96.6 \%)$ \\
Yes & \\
No & $25(52.1 \%)$ \\
Education Level & $12(45.8 \%)$ \\
High School & $1(2.1 \%)$ \\
College & \\
Post College & $31(58.5 \%)$ \\
Method of Migration & $12(22.6 \%)$ \\
Plane & $16(30.2 \%)$ \\
Boat & $14(26.4 \%)$ \\
Car & $18(34.0 \%)$ \\
Train/bus & \\
Walking & \\
\hline
\end{tabular}

sample of Bhutanese, Burmese, and Iraqi refugees (Hollifield et al. 2013). It is likely the advantages of Cuban entrants/refugees described in Rumbaut and Rumbaut (1976) are mitigating factors for mental health problems compared to other groups of refugees/entrants. Rumbaut and Rumbaut (1976) describes the Cuban exile as a "golden exile" because of the privileges which allow Cubans to adapt more easily such as being well-trained occupationally and academically, having a reason for exile which fits with American political values, access to a large Spanish-speaking community within the US, some familiarity with US culture in Cuba, and large Cuban communities which provide a comparison group and supportive network. The privileges described are consistent with what was found in our sample. Furthermore, past research states that the loyalty and family cohesion that are common to Cuban culture can be particularly protective (Bernal and Shapiro 2005). The hope for a better life in the US may lead to feelings of hopefulness and a lack of poor mental health outcomes in the months immediately after arrival. This idea is bolstered by previous studies that have found that refugee mental health decreases over time (Kirmayer et al. 2011). Cubans may adjust more easily to the US because of how they look, which may allow them to be perceived as White Americans compared to other groups of refugees and may limit the likelihood that they are perceived as different. Thus, Cubans may have less exposure to discrimination, racism, and xenophobic reactions, which have been shown to negatively impact mental health (Berger and Sarnyai 2015) and heighten culture shock (Shannon et al. 2016).
Overall, Cuban refugees/entrants from our sample appear to have better mental health than other groups of refugees.

\section{Limitations and Conclusion}

Limitations of the current study include its relatively small sample size and lack of inclusion of non-Cuban Latino participants. Additionally, the study relies on self-report measures and does not utilize multiple methods of reporting symptomatology such as structured diagnostic interviews. To address the limitations of this study and the need for more evidence for the validity and usefulness of the RHS-15, more research is needed. For example, future studies would benefit from recruiting larger samples of Cuban entrants/refugees and the use of structured clinical interviews to confirm a diagnosis in order to provide further evidence for the use of the RHS-15. Additionally, it would be advantageous to validate the RHS-15 in entrants/refugees of other Latino ethnicities and from other regions of the US in order to ensure the Spanish version's generalizability. Other future studies could examine the specific risk and protective factors that contribute to the poor mental health outcomes of the Cuban entrant/refugee population, attempt to determine if the treatment acceptance patterns found by Hollifield and colleagues (2013) (Hollifield et al. 2013) are present with Cuban entrants/refugees who are screened with the RHS-15, and examine the RHS-15 in comparison to other clinical interviews, to help understand the relatively low rates of psychopathology. 
Table 2 Mean scores for RHS-15 Items in Patients with Positive and Negative Screens

\begin{tabular}{lcccc}
\hline RHS-15 Items & $\begin{array}{c}\text { Negative RHS-15 } \\
\text { Screen } \\
\text { Mean (SD) }\end{array}$ & $\begin{array}{c}\text { Positive RHS-15 } \\
\text { Screen } \\
\text { Mean (SD) }\end{array}$ & t(df) & p \\
\hline 1. Muscle, bone, joint pains & $0.29(0.56)$ & $1.44(1.42)$ & $-2.39(8.55)$ & .04 \\
2. Feeling down, sad, or blue most of the time & $0.27(0.50)$ & $1.67(0.87)$ & $-4.68(9.21)$ & $<.01$ \\
3. Too much thinking/thoughts & $0.61(0.95)$ & $2.33(1.32)$ & $-4.60(48)$ & $<.01$ \\
4. Feeling helpless & $0.02(0.16)$ & $0.44(0.73)$ & $-1.73(8.16)$ & .09 \\
5. Suddenly scared for no reason & $0.02(0.16)$ & $0.22(0.67)$ & $-0.89(8.19)$ & .40 \\
6. Faintness, dizziness, or weakness & $0.07(0.26)$ & $0.44(0.73)$ & $-1.51(8.47)$ & .17 \\
7. Nervousness or shakiness inside & $0.17(0.54)$ & $0.67(0.87)$ & $-1.65(9.43)$ & .13 \\
8. Feeling restless, can't sit still & $0.24(0.49)$ & $0.78(1.09)$ & $-1.43(8.72)$ & .19 \\
9. Crying easily & $0.29(0.60)$ & $1.67(1.41)$ & $-2.86(8.65)$ & .02 \\
10. Reliving the trauma & $0.02(0.16)$ & $0.00(0.00)$ & $0.47(48)$ & .64 \\
11. Physical reactions when reminded of trauma & $0.00(0.00)$ & $0.44(1.01)$ & $-1.32(8)$ & .23 \\
12. Felt emotionally numb & $0.02(0.16)$ & $0.11(0.33)$ & $-0.76(8.79)$ & .47 \\
13. Jumpier, more easily startled & $0.05(0.22)$ & $0.89(1.36)$ & $-1.84(8.09)$ & .10 \\
14. Ability to cope & $0.32(0.52)$ & $0.89(0.78)$ & $-2.71(48)$ & .01 \\
15. Distress Thermometer & $1.29(1.12)$ & $6.11(1.54)$ & $110.89(48)$ & $<.01$ \\
RHS-15 Total Score & $2.41(2.49)$ & $12.00(3.84)$ & $7.16(9.53)$ & $<.01$ \\
\hline
\end{tabular}

Table 3 Principal Components Analysis

\begin{tabular}{lcccc}
\hline & \multicolumn{4}{c}{ Component } \\
RHS-15 Item & $\mathbf{1}$ & $\mathbf{2}$ & $\mathbf{3}$ & $\mathbf{4}$ \\
\hline 1. Muscle, bone, joint pains & .376 & .749 & -.171 & -.077 \\
2. Feeling down, sad, or blue most of the time & .300 & .401 & .224 & .467 \\
3. Too much thinking/thoughts & .042 & .381 & .740 & .298 \\
4. Feeling helpless & $\mathbf{. 8 0 1}$ & .137 & -.092 & .043 \\
5. Suddenly scared for no reason & .008 & .812 & -.040 & -.095 \\
6. Faintness, dizziness, or weakness & .778 & .018 & -.126 & .065 \\
7. Nervousness or shakiness inside & .720 & -.003 & .123 & -.122 \\
8. Feeling restless, can't sit still & $\mathbf{. 5 7 7}$ & -.178 & .524 & .184 \\
9. Crying easily & $\mathbf{. 5 5 0}$ & .481 & .256 & .012 \\
10. Reliving the trauma & -.043 & -.081 & .717 & -.180 \\
11. Physical reactions when reminded of trauma & -.242 &. $\mathbf{5 7 6}$ & .246 & .230 \\
12. Felt emotionally numb & -.140 & -.164 & -.070 & .772 \\
13. Jumpier, more easily startled & $\mathbf{. 5 9 2}$ & .227 & .264 & .130 \\
14. Ability to cope & $\mathbf{. 5 5 6}$ & -.027 & -.225 & .491 \\
15. Distress Thermometer & .284 & .443 & .310 & $\mathbf{. 5 8 4}$ \\
\hline Component loadings 0.550 or higher are shown in boldface. & & &
\end{tabular}

Overall, the RHS-15 has mounting evidence of its validity and clinical utility for refugees from a variety of countries, and this study provides generalizability information for its use with a Cuban population. The RHS-15 is an important clinical tool as it may allow for early identification, prevention, and treatment for incoming refugees who are experiencing psychopathology.

Acknowledgements: The authors would like to thank Judy Mier-Chairez and Ghazel Tellawi, MS, for assistance with data collection and Darlene Davis, MA, for early study planning. We also thank our volunteer clinical supervisors, including Virginia Frazier, PsyD, Eric Russ, PhD, and Gareth Holman, PhD.

Disclosure: The authors declare no conflict of interest.

\section{References}

Aspinall, Peter, and Charles Watters. 2010. Refugees and Asylum Seekers: A Review From an Equality and Human Rights Perspective. Manchester: Equality / Human Rights Commission.
Beck, Aaron T. 1990. Beck Anxiety Inventory. San Antonio, TX: Psychological Corporation.

Beck, Aaron T., Robert A. Steer, and Gary K. Brown. 1996. Manual for Beck Depression Inventory II (BDI-II). San Antonio, TX: Psychological Corporation.

Berger, Maximus, and Zoltán Sarnyai. 2015. "More than skin deep': Stress neurobiology and mental health consequences of racial discrimination." Doi:10.3109/10253890.2014.989204, Stress: The International Journal on the Biology of Stress 18 (1): 1-10.

Bernal, Guillermo, and Ester Shapiro. 2005. "Cuban families." In Ethnicity and family therapy, 3rd ed., edited by M. McGoldrick, J. Giordano, and N. Garcia-Prieto, 202-215. New York, NY: Guilford Publications, Inc. 
Table 4 Pearson's Correlations between the RHS-15 and measures of Anxiety, Depression, and PTSD

\begin{tabular}{lcccccc}
\hline & $\begin{array}{c}2 . \text { RHS-15 } \\
\text { total }\end{array}$ & $\begin{array}{c}\text { 3. BAI } \\
\text { therm. }\end{array}$ & $\begin{array}{l}\text { 4. BDI-II } \\
\text { 5. PCL-5 }\end{array}$ & 6. HSCL-25 & 7. MPSS-SR \\
\hline 1. RHS-15 total score & $.66^{* *}$ & $.56^{* *}$ & $.56^{* *}$ & $.49^{* *}$ & $.66^{* *}$ & $.46^{* *}$ \\
2. RHS-15 distress thermometer & - & $.58^{* *}$ & $.57^{* *}$ & $.51^{* *}$ & $.57^{* *}$ & $.36^{* *}$ \\
3. BAI & - & - & $.58^{* *}$ & $.86^{* *}$ & $.75^{* *}$ & $.81^{* *}$ \\
4. BDI-II & - & - & - & $.43^{*}$ & $.77^{* *}$ & $.58^{* *}$ \\
5. PCL-5 & - & - & - & - & $.76^{* *}$ & $.95^{* *}$ \\
6. HSCL-25 & - & - & - & - & - & $.90^{* *}$ \\
\hline
\end{tabular}

Note: $\quad$ RHS-15 = Refugee Health Screener 15, BAI = Beck Anxiety Inventory, BDI-II = Beck Depression Inventory, Second Edition, PCL-5 = Posttraumatic Stress Checklist- Civilian Edition, HSCL-25 = Hopkins Symptom Checklist-2, MPSS = Modified PTSD Symptom Scale.

$* *$ denotes significance at $.01, *$ denotes significance at .05

Bonicatto, Silvia, Amanda Mary Dew, and Juan Jose Soria. 1998. "Analysis of the psychometric properties of the Spanish version of the Beck Depression Inventory in Argentina." Psychiatry Research 79 (3): 277-285.

Bosson, Rahel, Monnica T. Williams, Stephen Lippman, Ruth Carrico, Jonathan Kanter, Analien Peña, Judy Mier-Chairez, and Julio A. Ramirez. 2017. "Addressing refugee mental health needs: From concept to implementation." Behavior Therapist 40 (3): 110-112.

CDC (Centers for Disease Control and Prevention). 2012a. General Refugee Health Guidelines. http : / / www . cdc.gov / immigrantrefugeehealth/pdf/general.pdf.

. 2012b. Guidelines and Discussion of the History and Physical Examination. http: //www. cdc.gov/immigrantref ugeehealth/pdf/guidelines-history-physical. pdf.

Costello, Anna B., and Jason W. Osborne. 2005. "Best practices in exploratory factor analysis: Four recommendations for getting the most from your analysis." Practical Assessment Research \& Evaluation 10 (7). http: / / pareonline. net / getvn. asp?v=10\&n=7.

Derogatis, Leonard R., Ronald S. Lipman, Karl Rickels, Eberhard H. Uhlenhuth, and Lino Covi. 1974. "The Hopkins Symptom Checklist (HSCL): A self-report symptom inventory." Doi:10.1002/bs.3830190102, Behavioral Science 19 (1): 115.

Falsetti, Sherry A., Heidi S. Resnick, Patricia A. Resick, and Dean G. Kilpatrick. 1993. "The Modified PTSD Symptom Scale: A brief self-report measure of posttraumatic stress disorder." The Behavioral Therapist 16:161-162.

Fierros, Melinda, and Christian Smith. 2006. "The relevance of Hispanic culture to the treatment of a patient with posttraumatic stress disorder (PTSD)." Psychiatry (Edgmont) 3:49.
Florida Department of Children and Families Refugee Services Program. 2015. "Cuban and Haitian Entrants." Chap. 3 in Refugee Program Eligibility Guide for Service Providers, 311. http : / / www. dcf . state. fl. us / programs / refugee/webguides/masterguide.pdf.

Foa, Edna B, Laurie Cashman, Lisa Jaycox, and Kevin Perry. 1997. "The validation of a self-report measure of posttraumatic stress disorder: The Posttraumatic Diagnostic Scale." Psychological Assessment 9:445-451.

Hollifield, Michael, Eric C. Toolson, Sasha Verbillis-Kolp, Beth Farmer, J. Yamazaki, Tsegaba Woldehaimanot, and Annette Holland. 2016. "Effective screening for emotional distress in refugees: The refugee health screener." Doi:10.1097/NMD.0000000000000469, Journal of Nervous and Mental Disease 204 (4): 247-253.

Hollifield, Michael, Sasha Verbillis-Kolp, Beth Farmer, Eric Toolson, Tsegaba Woldehaimanot, Junko Yamazaki, and Janet SooHoo. 2013. "The Refugee Health Screener15 (RHS-15): Development and validation of an instrument for anxiety, depression, and PTSD in refugees." Doi:10.1016/j.genhosppsych.2012.12.002, General Hospital Psychiatry 35:202-209.

Hollifield, Michael, Teddy D Warner, Barry Krakow, Janis Jenkins, and Joseph Westermeyer. 2009. "The range of symptoms in refugees of war: The New Mexico Refugee Symptom Checklist-121." Doi:10.1097/NMD.0b013e31819642dc, Journal of Nervous and Mental Disease 197 (2): 117-125.

Kirmayer, Laurence J, Lavanya Narasiah, Marie Munoz, Meb Rashid, Andrew G Ryder, Jaswant Guzder, Ghayda Hassan, Cécile Rousseau, and Kevin Pottie. 2011. "Common mental health problems in immigrants and refugees: general approach in primary care." Canadian Medical Association Journal 183 (12): E959-E967.

Krogstad, Jens Manuel. 2016. Surge in Cuban immigration to U.S. continues into 2016. Pew Research Center, August. http: // www. pewresearch.org/fact-tank/2016/08/05/cu ban-immigration-to-u-s-surges-as-relationswarm/. 
Magán, Inés, Jesús Sanz, and María Paz García-Vera. 2008. "Psychometric properties of a Spanish version of the Beck Anxiety Inventory (BAI) in general population.” The Spanish Journal of Psychology 11 (02): 626-640.

Marshall, Grant N. 2004. "Posttraumatic stress disorder symptom checklist: Factor structure and English-Spanish measurement invariance." Journal of Traumatic Stress 17 (3): 223-230.

Minnesota Department of Human Services. 2014. Verification I94 Cards. http: / / www. dhs. state.mn. us / main / id cplg? IdcService =GET_DYNAMIC_CONVERSION \& dDo cName $=$ cm_00101824\&RevisionSelectionMethod $=$ LatestReleased.

Orlando, Maria, and Grant N Marshall. 2002. "Differential item functioning in a Spanish translation of the PTSD checklist: detection and evaluation of impact." Psychological Assessment 14 (1): 50-59.

Parloff, Morris B., Herbert C. Kelman, and Jerome D. Frank. 1954. "Comfort, effectiveness, and self-awareness as criteria for improvement in psychotherapy." American Journal of Psychiatry 3:343-351.

Pathways to Wellness. 2012. Integrating refugee health and wellbeing. Creating pathways for refugee survivors to heal. Refugee Health Screener-15: Development and use of the RHS-15. htt $\mathrm{p}$ : //refugeehealthta.org/wp-content/uploads / 2012/09/RHS15_Packet_Pathways ToWellness-1. pdf.

Penley, Julie A, John S Wiebe, and Azikiwe Nwosu. 2003. "Psychometric properties of the Spanish Beck Depression Inventory-II in a medical sample." Psychological Assessment 15 (4): 569-577. https : / / doi .org/10 . 1037/10403590.15 .4 .569 .

Portes, Alejandro, David Kyle, and William W Eaton. 1992. "Mental illness and help-seeking behavior among Mariel Cuban and Haitian refugees in South Florida." Journal of Health and Social Behavior 33 (4): 283-298.

Pumariega, Andres J., Eugenio Rothe, and JoAnne B. Pumariega. 2005. "Mental health of immigrants and refugees." Community Mental Health Journal 41 (5): 581-597.
Refugee Processing Center. 2016. Top ten refugee native languages. http : / / www . wrapsnet . org/Reports / Admissions Arrivals/tabid/211/Default.aspx.

Rumbaut, Rubén D., and Rubén G. Rumbaut. 1976. "The family in exile: Cuban expatriates in the United States." American Journal of Psychiatry 133 (4): 395-399.

Shannon, Patricia, Hyojin Im, Emily Becher, Jennifer Simmelink, Elizabeth Wieling, and Ann O'Fallon. 2016. "Screening for war trauma, torture, and mental health symptoms among newly arrived refugees: A national survey of U.S. refugee health coordinators." Journal of Immigrant \& Refugee Studies 10 (4): 380-394.

Slonim-Nevo, Vered, and Shirley Regev. 2016. "Risk Factors Associated with Culture Shock among Asylum Seekers from Darfur." Journal of Refugee Studies 29 (1): 117-138.

UN General Assembly. 1951. Convention Relating to the Status of Refugees. http://www. unhcr. org/3b66c2aa10.html.

UNHCR (United Nations High Commissioner for Refugees). 2015. Global trends forced displacement in 2015. http: / / www . un hcr.org/en-us/statistics/unhcrstats/576408c d7/unhcr-global-trends-2015.html.

Ventevogel, Peter, Guglielmo Schinina, Alison Strang, Marcio Gagliato, and Louise Juul Hansen. 2015. "Mental health and psychosocial support for refugees, asylum seekers and migrants on the move across Europe: A multi-agency guidance note." www . euro . who . int / en / health topics / health - determinants / migration - andhealth/publications/2016/mental-health-andpsychosocial-support-for-refugees, - asylumseekers - and - migrants - on - the - move - in europe.-a-multi-agency-guidance-note-2015.

Weathers, Frank W., Brett T. Litz, Terence M. Keane, Patrick A. Palmieri, Brian P. Marx, and Paula P. Schnurr. 2013. The PTSD Checklist for DSM-5 (PCL-5). Scale available from the National Center for PTSD at www.ptsd.va.gov. 